\title{
Pre-symptomatic genetic testing for inherited cardiac conditions: a qualitative exploration of psychosocial and ethical implications
}

\author{
Elizabeth Ormondroyd ${ }^{\star, 1}$, Stephanie Oates ${ }^{2}$, Michael Parker ${ }^{3}$, Edward Blair ${ }^{4}$ and Hugh Watkins ${ }^{1}$ \\ Inherited cardiac conditions (ICCS) can lead to sudden cardiac death at any age, yet are often asymptomatic and clinically \\ undetected. Prophylactic interventions are available and cascade testing is recommended to identify family members at risk. \\ When a disease-causing mutation has been identified in a family, pre-symptomatic genetic testing (PSGT) is available. \\ This study explores perceptions of the cascade process, impact of PSGT and attitudes towards direct contact as an alternative \\ to family-mediated dissemination for ICCs. In depth, interviews were conducted with 22 participants eligible for PSGT for \\ Hypertrophic Cardiomyopathy or Long QT syndrome. Data were analysed using an inductive, thematic approach. Risk is \\ perceived to be low pre-test in the absence of symptoms, and participants frequently test with the aim of ruling out risk to \\ self and children. Testing of children is a complex decision; although older participants have concerns about possible adverse \\ effects of genetic testing early in the life course, young participants are pragmatic about their result. The meaning of a positive \\ genetic test result may be difficult to conceptualise in the absence of clinical evidence of disease, and this may deter further \\ dissemination to at-risk family members. A majority of participants see advantages in direct contact from health professionals \\ and support it in principle. Implications for practice include addressing risk perception pre-test, and presenting genetic test \\ information as part of a risk stratification process rather than a binary outcome. Families may require more support or \\ intervention in cascading genetic test information.
}

European Journal of Human Genetics (2014) 22, 88-93; doi:10.1038/ejhg.2013.81; published online 1 May 2013

Keywords: HCM; LQTS; genetic; psychosocial; qualitative

\section{INTRODUCTION}

Clinical genetic testing is now available for many inherited cardiac conditions (ICCs) including cardiomyopathies and ion channelopathies. Collectively affecting more than one person in 500, ICCs are understood to be single-gene autosomal-dominant conditions and are often asymptomatic, yet can present with cardiac arrhythmias and sudden cardiac death at any age. Disease expression within and between families is very variable and many pathogenic mutations have been shown to have incomplete penetrance. ICCs are genetically heterogeneous: many mutations in any one of several genes cause the same phenotype and knowledge of the specific mutation usually contributes little to risk stratification. ${ }^{1,2}$ These factors present considerable clinical, counselling and psychosocial challenges for patients, families and clinicians.

Evaluation of relatives in a step-wise manner (cascade testing) is recommended after an initial diagnosis with the aim of identifying asymptomatic individuals in the extended family who might be at the risk of disease-related complications. ${ }^{3}$ Affected individuals are offered lifestyle advice including avoidance of competitive sports, ${ }^{4}$ and evaluated for risk of sudden cardiac death and other disease complications. Pharmacological treatment is indicated for symptom relief and prophylaxis, and an implantable cardioverter defibrillator is effective for primary or secondary prevention of sudden cardiac death. ${ }^{5}$ Where a genetic diagnosis is available, pre-symptomatic genetic testing (PSGT) is a cost-effective means of identifying the $50 \%$ of relatives who are at risk and require cardiological evaluation, allowing discharge of the other 50\% who have not inherited the disease-causing allele. ${ }^{6,7}$ Among pre-symptomatically tested hypertrophic cardiomyopathy (HCM) gene carriers, around $33 \%$ go on to develop manifest HCM during follow up. ${ }^{8}$

When cascade testing relies on family-mediated contact, uptake of PSGT for HCM, at around $40 \%,{ }^{9}$ is similar to that for cancer predisposition; ${ }^{10}$ uptake is somewhat higher for channelopathies. ${ }^{11}$ Communication processes, and their impact on uptake of pre-symptomatic testing, have not been evaluated for ICCs.

Psychosocial research into pre-symptomatic/predictive testing to date, mainly focused on cancer predisposition and Huntington's Disease, shows that people undergoing PSGT do not experience adverse psychological consequences such as distress, anxiety or depression in the short term ${ }^{12,13}$ or the longer term ${ }^{14}$. There are several important differences between ICCs and other conditions for which PSGT is offered: (1) treatments are available for symptom control and prevention of SCD; (2) a positive ICC genetic test result in an adult can imply immediate risk irrespective of age; (3) ICCs can affect minor age children, ${ }^{1,15}$ whereas testing of children is considered with caution for 'later onset' conditions; ${ }^{16}$ (4) a positive PSGT result

${ }^{1}$ Department of Cardiovascular Medicine, University of Oxford, Level 6 West Wing, John Radcliffe Hospital, Oxford, UK; ${ }^{2}$ Genetic Health Service NZ-Northern Hub, Building 30 Auckland City Hospital, Auckland, New Zealand; ${ }^{3}$ The Ethox Centre, Department of Public Health, University of Oxford, Oxford, UK; ${ }^{4}$ Department of Clinical Genetics, Churchill Hospital, Oxford, UK

*Correspondence: Dr E Ormondroyd, Department of Cardiovascular Medicine, University of Oxford, Level 6 West Wing, John Radcliffe Hospital, Oxford OX3 9DU, UK Tel: +44 1865234676 or +44 7812 391582; Fax: + 441865 234677; E-mail: Liz.ormondroyd@cardiov.ox.ac.uk

Received 15 November 2012; revised 19 February 2013; accepted 13 March 2013; published online 1 May 2013 
for an ICC per se provides little information about the level of risk implied; (5) lifestyle/employment may be impacted. An understanding of these issues and the psychological impact of conferring genetic risk status on pre-symptomatic individuals is crucial for genetic counselling in ICCs, and for facilitating uptake of testing in relatives.

The aims of the present study were to explore the process of cascade genetic testing within families for either of the two most common ICCs, Long QT syndrome (LQTS) and HCM: to understand how people learn about risk and make decisions about undergoing PSGT, and to evaluate the psychosocial impact of such testing. A further specific aim was to explore attitudes to direct contact of relatives about the ICC and availability of PSGT. A qualitative methodology was chosen to elicit participants' experiences and perceptions.

\section{MATERIALS AND METHODS \\ Setting}

Genetic testing at our centre forms part of a multidisciplinary adult cardiology/ genetics (ICC) service, and is offered after counselling by a specialist genetic counsellor (EO, SO) or clinical geneticist (EB). Such specialised cardiac genetics clinics are highly satisfactory to HCM patients and relatives at risk of HCM. ${ }^{17,18}$ Mutation screening of selected genes is initially undertaken in a clinically affected individual, the 'proband'. When genetic test results become available, the proband is provided with a letter from our service, which can be passed to relatives, providing brief information about the condition and risks to relatives. Family members are counselled by a specialist genetic counsellor about the benefits, limitations and implications of PSGT. Genetically affected individuals are followed up thereafter in clinic.

\section{Participants}

Invitation letters were sent from HW to individuals who had undergone PSGT though Oxford University Hospitals Inherited Heart Conditions clinic since 2004, but not within the preceding 6 months, and were aged over 18 years at the time of invite. Purposive sampling was used to recruit a diverse group of participants with respect to gender, age and family history events relating to the ICC. Some participants had had cardiac evaluation before their genetic test. All participants who received a positive genetic test result had undergone subsequent cardiac evaluation as recommended.

\section{Recruitment}

Eligible participants were asked to return a reply slip providing preferred contact details, and contacted initially by telephone. Participants were asked if they would be willing to pass an invitation letter and information sheet to atrisk family members who were aware of the availability of genetic testing but had not sought genetic counselling. Written informed consent was taken before interview, and the study was approved by the Oxfordshire Research Ethics Committee (09/H0604/110).

\section{Interviews}

An interview guide was devised from the research questions: participants were asked to describe the history of heart problems in their family, how they learnt they might be at risk and reactions to learning about their risk, factors that influenced decisions about genetic testing, experiences and expectations of genetic counselling, whether/how the test result has influenced outlook on life, family relationships, lifestyle, employment choices, etc. and family communication. Direct contact was described by the interviewer. Participants were interviewed face to face by EO between April 2010 and July 2011. Interviews lasted $30 \mathrm{~min}$ to $1 \mathrm{~h}$ and were digitally recorded and transcribed verbatim with consent.

\section{Data analysis}

A thematic qualitative analysis of interview transcripts was undertaken using the method of constant comparison. ${ }^{19}$ This method involves systematically comparing interview transcripts in order to identify common themes and experiences. All transcripts were checked against interviews and initial codes made. A subset of transcripts was read by SO, MP, EB and EO and a list of broad categories generated. Transcripts were re-read in the light of this initial categorisation, a coding scheme developed and used to code the remaining transcripts (EO) and revised as analysis progressed. Higher order themes presented were derived from these emergent codes.

\section{RESULTS}

A total of 56 individuals were invited to participate (47 HCM, 9 LQTS) and 22 (18 HCM, 4 LQTS) consented to take part; response rate 39\%. There were no significant differences between responders and non-responders with respect to age, gender, clinical or genetic status. Three participants were willing to pass on letters to relatives (Table 1).

Analysis of the data generated four main themes: perception of risk, meaning of genetic test, coping with children's risk and communication of risk to the wider family.

\section{Perception of risk}

In some families, knowledge about the ICC was entwined with rumours, assumptions and uncertainties, which were investigated when a diagnosis was made or new relationship formed and the entrant to the family suggested their partner be checked. Perception of risk personally, and by extension to children, was not apparently heightened by the impact that the ICC had had on the family. Participants in whose families the ICC had caused premature deaths used a variety of mechanisms to justify their reasoning for believing that they or their children were not at risk of tragic complications, such as reluctance to link individual tragic events to the family diagnosis, or putting a distance between one branch of the family in which sudden deaths had occurred and 'their' branch:

'I think, really and truly, all the way through, I had thought it was on my step sister's [half sister's] side'

(female HCM gene positive)

or invoking alternative explanations for premature deaths, such as unhealthy lifestyle.

Although many participants spoke unprompted about the spectrum of ICC expression including sudden death, HCM/LQT was considered a relatively mild condition:

'It does appear touch wood, down the low end of things that can happen to you. It does seem to happen later on in life and it's not an out and out killer' (male HCM gene positive)

Table 1 Participant characteristics (participants had undergone PSGT for HCM or LQTS)

\begin{tabular}{lr} 
Age (years) & $N(\%)$ \\
\hline$<30$ & $5(23)$ \\
$31-60$ & $15(68)$ \\
$>60$ & $2(9)$ \\
Female & $13(60)$ \\
With minor aged children ${ }^{a}$ & $11(50)$ \\
With adult children & $3(14)$ \\
Genetic test positive & $17(77)$ \\
Family history of sudden cardiac death or cardiac arrest & $11(50)$
\end{tabular}

No participant had an implantable cardioverter defibrillator. One participant who had not sought PSGT was recruited through a participating relative.

a $<18$ Years at time of participant's genetic test. 
'I just thought, well I could develop it later in life but then I could develop a lot of things later in life'

(female HCM gene negative)

Absence of symptoms - sometimes combined with a sense of having led an 'active' life - reduced perception of risk of HCM before testing. One participant, undecided about genetic testing, described his thoughts about his risk:

'I felt fairly healthy and well and never even thought of being ill in any way whatsoever, so to be told you might have this hereditary illness was probably quite a shock at the time, but I am very much the sort of person who lives in the moment really and thinking that if I didn't feel any symptoms myself I wasn't going to rush to go' (male, family history HCM, untested)

So for a variety of reasons, many participants perceived their risk to be low, and entered the pre-symptomatic test process for the purpose of 'ruling out' the gene change.

\section{Meaning of genetic testing}

Some parents discussed the difficulty of not knowing how seriously to take a child's symptoms, and viewed the initial discovery of a disease causing genetic variant as helpful confirmation of a diagnosis:

'It is just the conciseness that someone has taken your [child's] blood and actually come along and said actually that gene there is actually not how it should be because it puts it to bed... you can get something to treat it hopefully to make it reasonably ok'

(LQTS gene negative; affected son)

For some, a genetic diagnosis 'proved' that the ICC in an affected person was hereditary, and provided the impetus for initiating cascade testing when no previous clinical screening had been suggested or taken up.

The predominant motivation for coming forward for pre-symptomatic testing was the 'need to know', personally and/or for their children. Non-parents were either young adults who had tested in response to perceived pressure from older relatives, albeit the value of testing was appreciated:

'Well I suppose it's better knowing if you've got something, if it's treatable...it was a sort of joint decision really I said yes I'd go but mum and dad said you're going anyway, so I didn't get much choice in it which is fair enough'

(male HCM gene positive)

or were older and had health concerns they felt might be explained by a genetic test.

Although many participants who have had a positive genetic test took a pragmatic view of their genetic diagnosis, for others it was problematic. Particularly, participants who had thought their risk low for one or more of the reasons described above, expressed shock when found to have the familial variant:

'Really and truly, I was absolutely horrified when it came back and said that I had it. I mean I really was horrified...the doctors told me I couldn't have it so I couldn't pass it on. It was a dreadful shock' (female HCM gene positive)

Some viewed a genetic test result as being able to tell 'if you will get it' as opposed to detectable disease being present at a particular time, providing an impetus to maintain a healthy weight, stay active and avoid strenuous activity.
However, some participants whose clinical screens, post positive genetic test, were normal suggested that cardiac tests are more meaningful and questioned the relevance of a genetic test. Some of these 'genotype positive, phenotype negative' people stated definitively that they, and/or children in the same situation, 'do not have a heart problem', and find a positive genetic test result difficult to conceptualise, particularly with regard to children who are also have no clinical signs of the ICC:

'I have this piece of knowledge, it makes little or no difference to physically how I am. So how helpful is it, it's not helpful is it in a way...should I have worried about J [son] having that information for the future and should I have thought about those things harder before sticking my arm out?'

(female HCM gene positive)

'You see, as far as I'm concerned, it's actually become a bit of a nuisance really, unless they, until they can tighten it down to a specific thing. I didn't realise how wide, just because you have it doesn't mean you're going to get it and that's the problem isn't it? Do you limit a child's capabilities because of knowing that..I honestly have no idea because once you know, it always at the back of your mind'

(female HCM gene positive)

Notwithstanding the perceived limitations of genetic testing, participants were satisfied with the cardiac genetics service, had confidence that they were receiving optimal care and were appreciative of research efforts to increase understanding of ICCs.

\section{Coping with children's risk}

For participants who were parents $(N=14$ including 11 with minor aged children), children's risk was often their primary concern. Some stated that personally the result did not matter, in the context of believing their personal risk of ICC complications low, of other health problems they live with and of having made most of their life decisions:

'I am more down the line and you feel that the children are just setting out..perhaps shed a few tears quietly but it's no good upsetting A [clinically affected son] and one had to remain positive for him but when you see his frustration and there's nothing you can do..' (female HCM gene positive)

For one person, anxiety for his child in the period between learning that the ICC was hereditary and getting his test result was described as being very difficult to manage:

'It was a very dark period, worried I could pass it down to my son who was in the under 7 s, racing around the field and I was thinking he may have to stop doing this...'

(male HCM gene negative)

Some articulated the view that a genetic diagnosis might have influenced their choice of activities had they had the option of genetic testing when they were young, especially if knowing about the risk might have influenced management:

'If I had been tested in my late teens to early $20 \mathrm{~s}$, and from the day I was born I have always had the gene, I have always had the strong family history [of SCD] and all the other things, if back then I had had that implant device put into me, I can pretty much say that most of the things I have done or managed to do, I may not have been able to'

(male HCM gene positive). 
Of nine participants with minor aged children who remained at risk after the parent's test result, five had tested their children by the time of interview, a decision made with the child and after discussion with the other parent. Decision making about testing children in the remaining four families was neither fixed nor clearly arrived at, with some parent participants asking the opinion of the interviewer, or about other families' decisions. Parents expressed the tension between needing to safeguard their child by maintaining an appropriate level of concern and restriction, while not having them 'lying around in cotton wool'; how to balance these competing stances was presented by all parents of dependent children as a dilemma. Concerns expressed by those who have not (yet) had their children tested included insurance and psychological concerns, and possible adverse effects on marriageabilty. Parents described concerns about appropriate children's activity levels, and discussed decision making in the context of their child's personality and proclivities, for example one person, whose son 'does not really like sports':

'He is a bit of a hypochondriac...having thought it seemed pretty logical for my son to have it [genetic test] then subsequently I didn't feel it was such a benefit to him at this stage to be worrying about it'. (female HCM gene positive)

Participants rationalised these concerns in different ways, for example, by managing a child's activity levels without testing and suggesting the option of testing his son without the child's knowledge. Age of the child seemed less a factor in test decision making. Parents whose children had been tested described difficulties in knowing how much to trust the child to abide by restrictions, and when the child's symptoms warranted further investigation. They perceived that their child had now adjusted well to their positive test result, even when it had been difficult for both child and parents to accommodate initially:

'He was ten. And he took it really badly, he was 'that's the end of my life isn't it mum' and I thought oh my god what have we done letting him have this test but he is so competitive...but now, 2 years down the line he is fine, he is allowed to do long distance running but he is not allowed to run to his maximum'

(female HCM gene positive)

Some of the younger participants had grown up knowing about the ICC in the family, and described their evolving understanding of their risk and their parents' concerns:

'[as a child] you can't choose for yourself but I think, in sort of like your parents' situation, it's probably better if you could have got it done because they wouldn't have had to worry so much'

(female HCM gene negative, no children)

Another young adult endorsed the view of parent participants that as a child he had resented being 'reined in' physically but identified and now understood his parents' anxiety and wish for him to be tested, and had adjusted well to his risk:

'I know my limits, I have been told that $75 \%$ is my maximum, I am quite comfortable I know what that is. I don't regret anything, I've stayed relatively fit and learnt other skills at the same time. The best of a bad situation really. Just get better at other things'. (male HCM gene positive)

\section{Communication of risk to the wider family}

Some participants felt that other relatives 'needed to know' about their ICC risk, and have informed family members. Participants were unanimously appreciative of having been told, by a relative, about their risk. However, for some, the complexities of family relationships and reluctance to cause unwarranted alarm added to uncertainty about telling relatives, and most participants know of relatives who have not been told. Telling relatives may present a dilemma, especially when they personally feel ambivalent about the genetic test:

'I don't know how to disseminate that kind of information, I don't know whether we should really. My cousin is the same age as I am and to my knowledge is perfectly well and there doesn't seem to be any reason to pop up and say, hey, perhaps this is a problem' (female HCM gene positive)

Resentment and estrangement were barriers to informing others, and several had not told elderly relatives because they considered it 'not worth the worry' for them.

Direct contact was positioned by the interviewer as an alternative when family communication had not been possible, but its advantages as a first-line approach were recognised, in the context of individual family relationships:

'I think it isn't perhaps something that should be left to the family to communicate because we all have our own perceptions as to whether $X$ or $Y$ is healthy or needs to have a test. How on earth do we know, when we are not qualified to make that decision'

(female HCM gene positive)

The majority were in favour of direct contact with the consent of the affected relative. Nuanced opinions were expressed, considering their family circumstances and envisaging their personal response to learning about risk from a third party:

'I think they have a right to know'.

I: 'can you imagine how you might have reacted to getting a letter [from health care provider caring for affected relative]'?

'I think I would have been far removed enough to not worry about it, but at least you have the knowledge. If you had the symptoms, you might think there is something in this. The onus is on them isn't it' (female HCM gene positive)

Some expressed the view that they would wonder why no one in the family had 'bothered' to tell them, whereas others felt they would appreciate third party contact if the alternative was to remain in ignorance. Some suggested that if they knew their estranged relative had consented to third party contact, this may be construed as an expression of affection and may 'help to build bridges'. Direct contact was also felt by some to have the potential to reinforce information already provided by family members, who felt the information had not been taken seriously enough and/or further disseminated to adult or minor children. Participants were aware of the potential for anxiety that such a letter might create, and felt that providing a point of contact and written information would be essential.

\section{DISCUSSION}

Our findings suggest several potential stalling points in the cascade process for HCM/LQTS in families where a pre-symptomatic genetic test is available. ${ }^{9}$ In addition to well described barriers to family communication, ${ }^{20}$ ambivalence about the specific value of the genetic test in terms of the lack of prognostic value may impede further dissemination. Relatives not known to be affected by HCM/LQTS perceive their risk to be low, and use various mechanisms to explain this perception even when the condition has had tragic consequences in their family. Despite 'knowing' that the condition can cause SCD, a 
majority of participants believe their own risk of adverse events is low in the absence of symptoms. For the one participant interviewed before engagement with our service, low personal risk perception was a key factor in choosing not to test, and we speculate that this is true for others who do not come forward for testing. In this context, individuals decide in favour of PSGT to rule out risk personally, and by extension to their children. When children are at risk, the decision to test is carefully deliberated and parents feel they make the decision in the context of the child's personality and activity levels. Parents of at-risk children - including those untested - worry about appropriate levels of activity and whether the child is abiding by restrictions. Some parents who have not (yet) tested their children are worried about the possible adverse psychological impact testing may have, and have concerns about restricting activity unnecessarily; however, young adults in the study who tested as children or adolescents are pragmatic about testing and a positive result. A majority of participants expressed favourable opinions regarding the possibility of direct contact in families with ICCs, with the agreement and cooperation of the family members seen in clinic.

The absence of symptoms impacts on risk perception in ICC, regardless of family history, and people who are 'genotype positive, phenotype negative' also perceive their risk as low. This agrees with earlier research that finds no difference in health-related quality of life between healthy HCM mutation carriers and the general population. ${ }^{18,21}$ It is likely that cardiac evaluation mitigates against any anxiety because of family history and/or PSGT result. Family history is a factor in lay perception of causation in cardiovascular (coronary) disease, although lifestyle is the major contributor, ${ }^{23}$ and it is of note in the current study that lifestyle factors are invoked by some participants to explain premature deaths.

Our findings with regard to parents' attitude to testing children accord with those of Geelen et $a l^{24}$ : parents try to make decisions in their children's best interests and the decision is a dynamic process constructed over time. In health-care decisions concerning children, it is widely agreed that the best interests of a child should be the primary consideration. ${ }^{25}$ As LQTS (and other channelopathies) can affect children at a young age and drug treatment is effective in reducing risk of serious events, pre-symptomatic testing of children for LQTS seems uncontroversial. Guidelines for screening children for HCM are so far lacking; our approach is to start screening of children at $50 \%$ risk around 8-10 years of age, or earlier if suspicious symptoms or family history of young onset disease are present. Current UK guidelines on PSGT in children state 'there may be good reasons to defer testing until such time when surveillance might be implemented, including to enhance the opportunity for the child to participate in discussions. ${ }^{16}$ However, it is likely that children of this age, while encouraged and often able to take part in the pre-symptomatic test process, are not fully able to comprehend the implications and arguably requests to test younger children should be considered.

An additional factor is the impact of ICC risk on lifestyle choices. Parents may be concerned that a child at $50 \%$ risk will develop an inclination for competitive sports, which will be unsustainable should HCM develop. Knowing that the child is at risk from a young age can be beneficial for advocating and encouraging alternative pastimes and presents a strong argument in favour of childhood testing. ${ }^{26}$

Parents in the present study contextualise decisions about testing children, taking into account the individual child's personality and activity levels. Some expressed concern about possible detrimental effects of a positive test result on their child in terms of causing the child unnecessary worry, or limiting their life prospects. Although we did not set out to address this specific issue or to recruit people who had been tested as children, our findings suggest that children and parents have adapted well to their test result and incorporate the result with clinical advice on lifestyle modifications with reasonable success. However, high levels of distress were reported in parents with children who tested positive for a LQTS mutation; ${ }^{27}$ fear of children dying, uncertainty, compliance with medication and lifestyle restrictions are concerns for parents of LQTS carrier children. ${ }^{28}$ Children who had a positive genetic test for an ICC (including HCM and LQTS; ${ }^{29}$ ) did not have significantly different quality-of-life scores than their peers, and apparently adapted well to their result. The long-term impact of pre-symptomatic testing on children and families for ICCs requires further research.

A positive genetic test result can be a threatening event; Biesecker and Erby $^{30}$ discuss adaptation to genetic risk in the context of Taylor's theory of cognitive adaptation. ${ }^{31}$ Three central components in the process of adaptation to threatening events are defined: a search for meaning, efforts to regain mastery (control) and restoration of selfesteem. We find that the meaning of a genetic diagnosis is relatively subjective and can change over time depending on results of clinical evaluation in members of a family. The meaning of a genetic diagnosis in a child may be very different because of the age-specific nature of risk in ICCs and its interaction with physical exertion. That risk in ICCs may be modified (reduced) by lifestyle factors may help individuals to regain a sense of control, and this is supported by our findings that participants described being motivated to adhere to lifestyle recommendations following a positive result. In children and young people, identifying alternative activities where necessary is likely to be an important feature in both expressing control and developing selfesteem.

Higher rates of uptake have been achieved following direct contact by health-care practitioners. ${ }^{32}$ A family-mediated approach preserves patient confidentiality and avoids possibly unwanted intrusion, but its success depends upon effective communication from health practitioner to patient (proband), and from patient to relatives, that relatives are at risk and that a predictive test is available. ${ }^{9}$ In the context of cancer predisposition, family communication is often a burden for the index case $^{33}$ and flawed: hampered by lack of social contact, family rifts and relationship breakdown the quality of information may be poor ${ }^{20}$ and compromised by perceived lack of authority of the informing relative. ${ }^{34}$ At-risk relatives are frequently left uninformed of conditions that are treatable or for which surveillance is available. With consent of the proband, direct contact has been used for Familial hypercholesterolaemia in the UK and the Netherlands and is accepted by family members, ${ }^{35}$ although a recent study finds that family members prefer indirect cascading. ${ }^{36}$ Familial hypercholesterolaemia differs from HCM and LQTS in that diagnosis, assessment of risk and treatment are relatively uncomplicated and the disease process (coronary heart disease) widely known and understood. Ethical considerations are inherent to both approaches. ${ }^{37}$ Our findings that most relatives would accept - and envisage benefits in addition to alerting people to likely preventable risks - add to this debate and will be pursued.

\section{Limitations}

We do not suggest that these findings are generalisable; as a qualitative study, we aimed rather to explore in depth the psychosocial issues associated with decisions about PSGT. Our purposive sample was successful in reaching a diverse group with respect to age, gender and cardiac event history, but included few people who had tested for LQTS. Although representative of numbers attending our clinic, the study did not provide explanations to why cascading in LQTS is more successful than in HCM (11). From clinical practice, we are aware of a 
Table 2 Clinical issues and implications for relatives at risk of $\mathrm{HCM}$ or LQT

\begin{tabular}{ll} 
Clinical issue & Implication \\
\hline $\begin{array}{l}\text { Variability in clinical } \\
\text { presentation }\end{array}$ & $\begin{array}{l}\text { Possibility of positive GT result, with or without } \\
\text { disease expression, even if asymptomatic } \\
\text { Disease expression in one person does not predict in } \\
\text { others } \\
\text { Reduced penetrance }\end{array}$ \\
$\begin{array}{l}\text { Genetic diagnosis does not inform whether/when } \\
\text { person may experience symptoms or require } \\
\text { treatment }\end{array}$ \\
$\begin{array}{l}\text { Risk of complications and lifestyle recommenda- } \\
\text { tions will be determined by individual cardiac } \\
\text { surveillance }\end{array}$ \\
$\begin{array}{l}\text { surveillance } \\
\text { Leisure, employment be impacted } \\
\text { Lifestyle }\end{array}$ \\
Risk to relatives \\
adult relatives
\end{tabular}

small number of individuals who undergo PSGT and do not attend for clinical follow up; although eligible, they are not represented in the current study. We attempted to recruit individuals who had not sought genetic counselling but with one exception, this strategy was not successful. The majority of participants believed that all at-risk relatives had either been told and sought PSGT, or had not been told, or had been told but had declined testing and participant's opinion was that it was not appropriate to contact them for the purpose of the study.

\section{Implications for practice}

Findings presented highlight barriers to at-risk relatives being informed and/or coming forward for PSGT in ICCs. Issues relevant to at-risk family members are presented in Table 2 - it may be helpful if these messages are conveyed to the proband, in cascade information from ICC services, and discussed during PSGT counselling.

A family history of tragic complications may be moderated by alternative beliefs about causation and/or inheritance, which may have a valuable psychological role in helping at-risk individuals to cope with risk both personally and for their children. It may be important to preserve these beliefs following a positive PSGT result. PSGT counselling for HD and cancer presents a binary outcome, as a positive result confers high or complete risk of being affected. In ICCs, given the lack of clear prognostic value of genetic testing, it is more accurate, and possibly helpful to counsellees in their attribution of meaning, to present genetic testing as a first stage in a risk stratification process, emphasising the importance of cardiac evaluation after a positive genetic test result.

\section{CONFLICT OF INTEREST}

The authors declare no conflict of interest.

\section{ACKNOWLEDGEMENTS}

We thank the participants for their time. We are grateful to Kathryn Smith and Eunice Berry for transcribing the interviews. The study was made possible by a grant from the Oxford NIHR Biomedical Research Centre to HW and EB.

1 Goldenberg I, Moss AJ.: Long QT syndrome. J Am Coll Cardiol 2008; 51: 2291-2300. 2 Watkins H, Ashrafian H, Redwood C: Inherited cardiomyopathies. N Engl J Med 2011; 364: 1643-1656.

3 Heart Rhythm UK Familial Sudden Death Syndromes Statement Development Group. Clinical indications for genetic testing in familial sudden cardiac death syndromes: an HRUK position statement. Heart 2008; 94: 502-507.

4 Pelliccia A, Fagard R, Bjørnstad $\mathrm{HH}$ et al: Recommendations for competitive sports participation in athletes with cardiovascular disease: a consensus document from the Study Group of Sports Cardiology of the Working Group of Cardiac Rehabilitation and Exercise Physiology and the Working Group of Myocardial and Pericardial Diseases of the European Society of Cardiology. Eur Heart J. 2005; 26: 1422-1445.
5 Maron BJ.: Risk stratification and role of implantable defibrillators for prevention of sudden death in patients with HCM. Circ J. 2010; 74: 2271-2282.

6 Wordsworth S, Leal J, Blair E et al: DNA testing for hypertrophic cardiomyopathy: a cost-effectiveness model. Eur Heart J. 2010; 31: 926-935.

7 Ingles J, McGaughran J, Scuffham PA et al: A cost-effectiveness model of genetic testing for the evaluation of families with hypertrophic cardiomyopathy. Heart 2012; 98: 625-630.

8 Christiaans I, Birnie E, Bonsel GJ et al: Manifest disease, risk factors for sudden cardiac death, and cardiac events in a large nationwide cohort of predictively tested hypertrophic cardiomyopathy mutation carriers: determining the best cardiological screening strategy. Eur Heart J. 2011; 32: 1161-1170.

9 Christiaans I, Birnie E, Bonsel GJ et al: Uptake of genetic counselling and predictive DNA testing in hypertrophic cardiomyopathy. Eur J Hum Genet 2008; 16: 1201-1207.

10 Brooks L, Lennard F, Shenton A et al: BRCA1/2 predictive testing: a study of uptake in two centres. Eur J Hum Genet 2004; 12: 654-662.

11 van der Roest WP, Pennings JM, Bakker M et al: Family letters are an effective way to inform relatives about inherited cardiac disease. Am J Med Genet A. 2009; 149A: 357-363.

12 Broadstock M, Michie S, Marteau T.: Psychological consequences of predictive genetic testing: a systematic review. Eur J Hum Genet 2000; 8: 731-738.

13 Heshka JT, Palleschi C, Howley $\mathrm{H}$ et al: A systematic review of perceived risks, psychological and behavioral impacts of genetic testing. Genet Med 2008; 10: 19-32.

14 Foster C, Watson M, Eeles R et al: Predictive genetic testing for BRCA1/2 in a UK clinical cohort: three-year follow-up. Br J Cancer 2007; 96: 718-724.

15 Ostman-Smith I, Wettrell G, Keeton B et al: Age- and gender-specific mortality rates in childhood hypertrophic cardiomyopathy. Eur Heart J. 2008; 29: 1160-1167.

16 Report on the Genetic Testing of Children, British Society for Human Genetics 2010. http://www.bshg.org.uk/GTOC Booklet Final new.pdf, accessed on 25 June 2012

17 Ingles J, Lind JM, Phongsavan P, Semsarian C.: Psychosocial impact of specialized cardiac genetic clinics for HCM. Genet Med 2008; 10: 117-120.

18 Christiaans I, van Langen IM, Birnie E et al: Genetic counseling and cardiac care in predictively tested hypertrophic cardiomyopathy mutation carriers: the patients' perspective. Am J Med Genet A. 2009; 149A: 1444-1451.

19 Strauss A, Corbin J.: Basics of Qualitative Research Techniques and Procedures for Developing Grounded Theory, 2nd edn London: Sage Publications, 1998.

20 Forrest K, Simpson SA, Wilson BJ et al: To tell or not to tell: barriers and facilitators in family communication about genetic risk. Clin Genet 2003; 64: 317-326.

21 Ingles J, Yeates L, Hunt L et al: Health status of cardiac genetic disease patients and their at risk relatives. Int J Cardiol 2011; e-pub ahead of print 17 September 2011.

22 Christiaans I, van Langen IM, Birnie E et al: Quality of life and psychological distress in hypertrophic cardiomyopathy mutation carriers: a cross-sectional cohort study. Am J Med Genet A 2009; 149A: 602-612.

23 Hunt K, Davison C, Emslie C, Ford G.: Are perceptions of a family history of heart disease related to health-related attitudes and behaviour? Health Educ Res 2000; 15: 131-143.

24 Geelen E, Van Hoyweghen I, Doevendans PA et al: Constructing 'best interests': genetic testing of children in families with hypertrophic cardiomyopathy. Am J Med Genet A 2011; 155A: 1930-1938.

25 Borry P, Evers-Kiebooms G, Cornel MC et al: Public and Professional Policy Committee (PPPC) of the European Society of Human Genetics (ESHG). Genetic testing in asymptomatic minors: background considerations towards ESHG Recommendations. Eur J Hum Genet 2009; 17: 711-719.

26 Watkins H, Seidman JG, Seidman E.: Genetic Testing for Hypertrophic Cardiomyopathy. N Engl J Med 1992; 327: 1175-1176.

27 Hendriks KS, Grosfeld FJ, Wilde AA et al: High distress in parents whose children undergo predictive testing for long QT syndrome. Community Genet 2005; 8: 103-113.

28 Farnsworth MM, Fosyth D, Haglund C, Ackerman MJ.: When I go in to wake them... I wonder: parental perceptions about congenital long QT syndrome. J Am Acad Nurse Pract 2006; 18: 284-290.

29 Smets EM, Stam MM, Meulenkamp TM et al: Health-related quality of life of children with a positive carrier status for inherited cardiovascular diseases. Am J Med Genet A. 2008; 146A: 700-707.

30 Biesecker BB, Erby L: Adaptation to living with a genetic condition or risk: a mini-review. Clin Genet 2008; 74: 401-407.

31 Taylor S.E.: Adjustment to threatening events: A theory of cognitive adaptation. Am Psychol 1983; 38: 1161-1173.

32 Suthers GK, Armstrong J, McCormack J, Trott D.: Letting the family know: balancing ethics and effectiveness when notifying relatives about genetic testing for a familial disorder. J Med Genet 2006; 43: 665-670.

33 Hallowell N, Foster C, Eeles R et al: Balancing autonomy and responsibility: the ethics of generating and disclosing genetic information. J Med Ethics 2003; 29: 74-79.

34 Ormondroyd E, Moynihan C, Ardern-Jones A et al: Communicating genetics research results to families: problems arising when the patient participant is deceased. Psychooncology 2008; 17: 804-811.

35 van Maarle MC, Stouthard ME, Marang-van de Mheen PJ et al: How disturbing is it to be approached for a genetic cascade screening programme for familial hypercholesterolaemia? Community Genet 2001; 4: 244-252.

36 Hallowell N, Jenkins N, Douglas $M$ et al: Patients' experiences and views of cascade screening for familial hypercholesterolemia (FH): a qualitative study. J Community Genet 2011; 2: 249-257.

37 Newson AJ, Humphries SE.: Cascade testing in familial hypercholesterolaemia: how should family members be contacted? Eur J Hum Genet 2005; 13: 401-408. 